\title{
AN ANALYTICAL APPROXIMATION OF THE TRANSIENT RESPONSE OF A VOLTAGE DEPENDENT SUPERCAPACITOR MODEL
}

\author{
Tomislav Barić - Hrvoje Glavaš - Ružica Kljajić
}

Faculty of Electrical Engineering, Computer Science and Information Technology Osijek Josip Juraj Strossmayer University of Osijek, Kneza Trpimira 2b, 31000 Osijek

\begin{tabular}{l} 
ARTICLE INFO \\
\hline Article history: \\
Received: 21.9 .2018$. \\
Received in revised form: 15.01 .2019$. \\
Accepted: 23.01 .2019$. \\
\hline Keywords: \\
Differential equation \\
Supercapacitor \\
Ultracapacitor \\
Voltage dependent model \\
\hline DOI: http://doi.org/10.30765/er.39.3.12
\end{tabular}

\section{Introduction}

Supercapacitors (SCs), also known as ultracapacitors or electric double-layer capacitors (EDLCs), are names for energy storage devices which store energy

\begin{abstract}
:
Supercapacitors are well known for their voltage dependent capacity. Due to this, it is not possible to obtain the exact analytical solution of the nonlinear differential equation which describes the transient charging and discharging. For this reason, approximations of differential equations must be carried out in order to obtain an approximate analytical solution. The focus of this paper is on a different approach. Instead of approximating the differential equation and obtaining analytical expressions for such approximations, an intuitive approach is chosen. This approach is based on the separation of the initial response from the rest of the transient phenomenon. Both parts of the transient phenomenon are described with adequate functions. Using appropriate weight functions, both functions are combined into a single function that describes the whole transient phenomenon. As shown in the paper, such an approach gives an excellent description of the whole transient. Also, it provides simpler expressions compared to those obtained by approximation of the nonlinear differential equation. With respect to their accuracy, these expressions do not lag behind the aforementioned approach. The validity of the presented analytical expressions was confirmed by comparing their results with those obtained by numerically solving the nonlinear differential equation.
\end{abstract}

via a physical process of charge separation in the solid-liquid interface [1-6]. Unlike ordinary capacitors, SCs have several properties that make them particularly distinct. They have very pronounced characteristics: self-discharge, dielectric

\footnotetext{
*Corresponding author. Tel.:031 224 600; fax: 031224605

E-mail address: hrvoje.glavas@ferit.hr.
} 
absorption, and voltage dependent capacitance. Considering the range of capacitance change, i.e. when they are completely empty and are fully charged at rated voltage, changes in its capacity can be up to 50\% [7-8] and higher [9]. If the SCs are used in applications where its voltage changes in a wide range, this change of capacitance must be taken into account. Voltage dependent SC models are used for modeling such cases. In this case, the dynamic behavior of the SC is described by a nonlinear differential equations (NDE).

Unfortunately, this NDE is of a form in which it is not possible to obtain the exact analytical solution that describes the charging and discharging transient. For this reason, approximations of this NDE must be carried out in order to obtain an approximate analytical solution. Since such approximations are highly individualized, there are various forms of such approximations. Also, their accuracy varies greatly from case to case. Usually, most of them only serve for qualitative considerations. Accurate analysis is carried out with computer simulations in which the $\mathrm{SC}$ voltage dependent model is used.

Due to the existing need for accurate analytical expressions that describe the transient in the abovementioned cases, in this paper a different approach for obtaining this is chosen. Instead of approximating the differential equation and obtaining analytical expressions for such approximations, an intuitive approach is chosen. This approach is based on the separation of the initial response from the rest of the transient phenomenon. Each part of the transient phenomenon is well described with an adequate function. Using the appropriate weight functions, both functions are combined into a single function that describes the whole transient phenomenon. This paper demonstrates that for a given regime such as rapid charging/discharging, such an approach gives an excellent description of the whole transient. Also, it gives simpler expressions compared to those obtained by approximation of the NDE. Regarding their accuracy, these expressions do not lag behind the aforementioned approach. The validity of the presented analytical expressions is confirmed by comparing their results with those obtained by numerically solving the NDE.

\section{Problem description}

Several criteria should be considered simultaneously when determining a suitable approximate analytical expression. The analytical expression should be relatively simple, to a certain accuracy, with clear limits of its validity and, if possible, should have an elegant form that is in some way associated with the expressions obtained by using a voltage-independent model. Also, it may be desirable if the integral of such an expression can be determined. In this paper, exponential functions were chosen for weighting functions because of their elegance. They are used to separate the initial part of the transient from the remainder of the transient phenomenon. This way, each part of the transient is described by its own exponential function that is obtained in the traditional way. The proposed approximate expressions are associated with well-known expressions by which transient behaviour is described using the voltageindependent model.

Since exponential functions are selected as weight functions, their combination with exponential functions describing transients form a function for which an integral can be determined. This property is needed for determining energy losses in SC and circuits by integrating the current or its square. Otherwise, energy-related physical quantities must be determined in a different way, which is also shown in this article.

\section{The properties of SCs and its models}

Unlike ordinary capacitors, SCs have several properties that make them particularly distinct. They have very pronounced characteristics: self-discharge, dielectric absorption, and voltage dependent capacitance. There are numerous SC schemes by which the SC properties can be modeled [2, 7, 1016]. They can be arbitrarily divided into two categories. One, in which topology faithfully reflects the physical processes (physical models [10]) and their relationships that take place in SC. To build such schemes it is necessary to know the physical quantities within the SC, the physical processes and their relationships. Such schemes are suitable for analyzing the influence of internal parameters on external SC behavior. The other category includes schemes in which the topology is determined by appropriate equivalents [10]. The equivalents are such that the SC on its external terminals behaves in a way as is determined by external measurements. For their construction, it is not necessary to know the physical quantities within the SC, the physical processes, and their relationships. It is only necessary to know external SC behavior. The second approach is more popular among engineers. One such scheme in which the voltage dependent capacitance, 
dielectric absorption and self-discharge is taken into consideration is shown in Fig. 1.

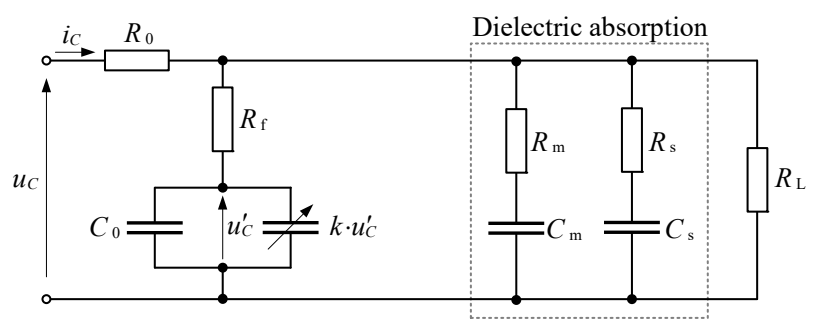

Figure 1.Voltage dependent supercapacitors equivalent scheme for the description of dynamic and static behaviour.

Regarding the speed (dynamics) of the process that takes places in the SC, the branch with capacitances $C_{0}$ and $k \cdot u_{\mathrm{c}}^{\prime}$ corresponds to fast process and the parallel RC branches by which dielectric absorption is modeled correspond to moderate and slow processes. Decomposition of SC capacitance into fast, moderate and slow process according to the scheme in Fig. 1. is not strictly defined. On the other hand, this gives a certain freedom in modeling. Resistance of the electrode and electrolyte material is taken into account by resistances $R_{0}$ and $R_{\mathrm{f}}[17,18]$. Self-discharge $[2,7,11]$ is modeled by a leakage resistance $R_{\mathrm{L}}$.

Dependence of SC capacitance upon the applied voltage is linear, but its variation from a state when $\mathrm{SC}$ is uncharged and fully charged at rated voltage can be up to $50 \%$ [7-8], and even higher [9].

Because SC capacitance is voltage dependent, the rated capacitance $C_{\mathrm{r}}$ is defined as capacitance at the rated voltage $U_{\mathrm{r}}$, and voltage dependence of the SC capacitance is usually approximated as follows

$$
C\left(u_{C}^{\prime}\right)=C_{0}+k \cdot u_{C}^{\prime}
$$

$C_{0}$ is the capacitance at zero voltage and $k$ is a coefficient that takes into account the capacitance variation with respect to voltage. It is defined by:

$$
k=\frac{C_{\mathrm{r}}-C_{0}}{U_{\mathrm{r}}}
$$

\section{The mathematical description of the SC charging}

If the model, according to Fig. 1, is made so that the time constants of the individual branches differ by an order of magnitude, then the branch with the voltage dependent capacitance has a dominant influence on the dynamic behavior in a rapid charge/discharge regime. That is, the branches which model dielectric absorption can be neglected. According to this assumption, if necessary, the effect of dielectric absorption, may be subsequently taken into account by using superposition. Therefore, the scheme shown in Fig. 2. is adopted to represent the equivalent model of SC in rapid charge/discharge regime.

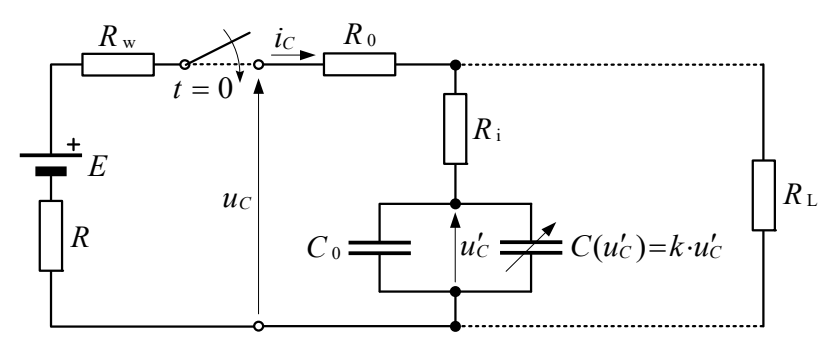

Figure 2. Equivalent scheme of an SC and external circuit for describing the rapid charge of supercapacitor. $\left(R_{\mathrm{w}}\right.$ denotes wire resistance).

After the completion of transients $(t \rightarrow \infty)$, voltage on the SC is determined by a voltage divider formed by the resistances: $R, R_{\mathrm{w}}, R_{0}$ and $R_{\mathrm{L}}$, and it reads as follows:

$$
u_{C}(\infty)=E \frac{R_{\mathrm{L}}}{R_{\mathrm{L}}+R_{0}+R_{\mathrm{W}}+R}=E \frac{R_{\mathrm{L}}}{R_{\mathrm{eqv}}}
$$

Where $R_{\text {ekv }}$ is equivalent to series resistance.

$$
R_{\mathrm{eqv}}=R+R_{\mathrm{w}}+R_{0}+R_{\mathrm{i}}
$$

Considering that $R_{\mathrm{L}}$ is several orders of magnitude higher than $R, R_{\mathrm{w}}$ and $R_{0}$, it can be assumed that

$$
u_{C}(\infty)=E
$$

During the transient, leakage current can be neglected, i.e. $R_{\mathrm{L}}$ can be excluded from further considerations. The application of Kirchhoff's Voltage Law to the scheme shown in Fig. 2 gives

$$
E-i_{C}\left(R_{0}+R_{\mathrm{W}}+R+R_{\mathrm{i}}\right)-u_{C}^{\prime}=0
$$

The current through SC is defined by the expression 


$$
i_{C}=\frac{\mathrm{d} q_{C}}{\mathrm{~d} t}
$$

Separated charge $q_{C}$ is related to SC voltage by the relation

$$
q_{C}=C\left(u_{C}^{\prime}\right) \cdot u_{C}^{\prime}
$$

Substituting (1) into (8) and applying (7) gives

$$
i_{C}=\frac{\mathrm{d}}{\mathrm{d} t} C\left(u_{C}^{\prime}\right) \cdot u_{C}^{\prime}=\left(C_{0}+2 \cdot k \cdot u_{C}^{\prime}\right) \frac{\mathrm{d} u_{C}^{\prime}}{\mathrm{d} t}
$$

Substituting (2), (4) and (9) into (6) gives

$$
R_{\mathrm{eqv}} \cdot\left(C_{0}+2 \cdot k \cdot u_{C}^{\prime}\right) \frac{\mathrm{d} u_{C}^{\prime}}{\mathrm{d} t}+u_{C}^{\prime}=E
$$

By introducing the following substitutions

$$
\begin{gathered}
a=R_{\mathrm{eqv}} \cdot C_{0} \\
b=R_{\mathrm{eqv}} \cdot 2 \cdot \frac{C_{\mathrm{r}}-C_{0}}{U_{\mathrm{r}}}
\end{gathered}
$$

expression (10) can be written in a concise form

$$
\left(a+b \cdot u_{C}^{\prime}\right) \frac{\mathrm{d} u_{C}^{\prime}}{\mathrm{d} t}+u_{C}^{\prime}=E
$$

Expression (13) is a first order NDE. The corresponding homogeneous differential equation is given by:

$$
\left(a+b \cdot u_{\mathrm{Ch}}^{\prime}\right) \frac{\mathrm{d} u_{\mathrm{Ch}}^{\prime}}{\mathrm{d} t}+u_{\mathrm{Ch}}^{\prime}=0
$$

Separation of variables in (14) gives:

$$
\frac{a+b \cdot u_{C h}^{\prime}}{u_{C h}^{\prime}} \mathrm{d} u_{C h}^{\prime}+\mathrm{d} t=0
$$

Integrating the previous expression gives:

$$
a \cdot \ln u_{\mathrm{Ch}}^{\prime}+b \cdot u_{\mathrm{Ch}}^{\prime}+t+\ln K=0
$$

Where $K$ is the integration constant determined from the initial conditions.
When $b \neq 0$, it is not possible to express the voltage $u_{\mathrm{Ch}}^{\prime}$ explicitly as a function of time $t$. For this reason, the NDE (13) does not have an exact analytical solution in which voltage $u_{\mathrm{Ch}}^{\prime}$ is explicitly described as a function of time. However, the approximate solution can be obtained by implementing different approximations that allow the voltage $u_{\mathrm{Ch}}^{\prime}$ to be explicitly expressed as a function of time and thereby voltage $u_{c}^{\prime}$. Instead of such an approach, an intuitive approach in determining the approximate analytical expression describing SCs voltage and current transients in rapid charge/discharge regime can be used. This approach is no less valuable than the previous one, since both approaches provide only an approximate expression which describes the transient.

\subsection{An intuitive approach in description of SCs transients}

By neglecting the member that describes the voltage dependent capacitance $(b=0), \quad$ a linear nonhomogeneous differential equation is obtained:

$$
a \frac{\mathrm{d} u_{C}^{\prime}}{\mathrm{d} t}+u_{C}^{\prime}=E
$$

which has a solution of the corresponding homogeneous differential equation (Eq. (16) for $b=0)$ in the form:

$$
a \ln u_{\mathrm{Ch}}^{\prime}+t+\ln K=0
$$

where $K$ is integration constant.

The previous expression can be written in the form:

$$
\ln \frac{u_{\mathrm{Ch}}^{\prime}}{K^{\prime}}=-\frac{t}{a}
$$

where $K^{\prime}=1 / K$. The antilogarithm of (19) gives:

$$
u_{\mathrm{Ch}}^{\prime}=K^{\prime} \cdot \mathrm{e}^{-\frac{t}{a}}
$$

Coupling of the homogeneous solution with the particular solution $\left(u_{C}^{\prime}=u_{C \mathrm{~h}}^{\prime}+u_{C \mathrm{p}}^{\prime}\right.$, where $\left.u_{C \mathrm{p}}^{\prime}=E\right)$ gives

$$
u_{C}^{\prime}=K^{\prime} \cdot \mathrm{e}^{-\frac{t}{a}}+E
$$


For $t=0$, it follows from (21) that $K^{\prime}=u_{C}^{\prime}(0)-E$. Introducing the substitution $\Delta U_{\max }=E-u_{C}^{\prime}(0)$ gives

$$
K^{\prime}=-\Delta U_{\max }
$$

Substituting (22) into (21) gives

$$
u_{C}^{\prime}=-\Delta U_{\max } \cdot \mathrm{e}^{-\frac{t}{a}}+E
$$

Usually, the constant $a$ is called time constant, denoted by $\tau$ or $T$. Since it is determined with the value of the capacitance $C_{0}$ (Eq. 11) when a SC is not charged, the notation $T_{0}$ is used in this paper. Accordingly, the expression (23) can be written in the form

$$
u_{C}^{\prime}=-\Delta U_{\max } \cdot \mathrm{e}^{-\frac{t}{T_{0}}}+E
$$

The member in brackets in Eq. (13) can be identified with a time constant which is voltage-dependent $T\left(u_{C}^{\prime}\right)$. Accordingly, Eq. (13) can be written in the form

$$
T\left(u_{C}^{\prime}\right) \frac{\mathrm{d} u_{C}^{\prime}}{\mathrm{d} t}+u_{C}^{\prime}=E
$$

Voltage-dependent time constant can be written in the form

$$
T\left(u_{C}^{\prime}\right)=R_{\mathrm{eqv}}\left(C_{0}+2 \frac{C_{\mathrm{r}}-C_{0}}{U_{\mathrm{r}}} u_{C}^{\prime}\right)
$$

Knowledge that the time constant changes during the transient phenomenon, and that we know its particular value at the beginning and the end of the transient, gives the idea how to describe transient by two expressions in the form of expression (24). One, for the initial part of the transient described by initial time constant $T_{\mathrm{i}}$, and another one for the rest of the transient phenomenon described by time constant near the end of transient $T_{\mathrm{e}}$. Transition from one expression to another should be smooth by the prescribed dynamics. For this purpose, two appropriate weight functions denoted as $w_{1}$ and $w_{2}$ can be used.

$$
\begin{aligned}
u_{C}^{\prime}= & w_{1}\left(-\Delta U_{\max } \mathrm{e}^{-\frac{t}{T_{\mathrm{i}}}}+E\right)+ \\
& +w_{2}\left(-\Delta U_{\max } \mathrm{e}^{-\frac{t}{T_{\mathrm{e}}}}+E\right)
\end{aligned}
$$

Since $w_{1}+w_{2}=1, \forall t$, Eq. (27) can be written in the following form

$$
u_{C}^{\prime}=-\Delta U_{\max } \cdot\left(w_{1} \cdot \mathrm{e}^{-\frac{t}{T_{\mathrm{i}}}}+w_{2} \cdot \mathrm{e}^{-\frac{t}{T_{\mathrm{e}}}}\right)+E
$$

Although Eq. (28) is mathematically correct, we know that only near the end of transient will it have the dynamics described by the time constant $T_{\mathrm{e}}$. For this reason, after the initial part ends, it is better to describe the rest of transient by time constant $T_{\mathrm{r}}$ (index "r" denotes "rest") which is somewhere between initial $T_{\mathrm{i}}$ and $T_{\mathrm{e}}$. In this paper $T_{\mathrm{r}}=0.5\left(T_{\mathrm{i}}+T_{\mathrm{e}}\right)$ has been selected.

For construction of weight functions $w_{1}$ and $w_{2}$ that ensure the separation of the initial part from the rest of the transient phenomenon, exponential functions [19] are selected. The weight functions $w_{1}$ and $w_{2}$ are of the form

$$
\begin{gathered}
w_{1}=\mathrm{e}^{-c \frac{t}{T_{\mathrm{i}}}} \\
w_{2}=1-\mathrm{e}^{-c \frac{t}{T_{\mathrm{i}}}}
\end{gathered}
$$

where the coefficient $c$ serves to adjust proper transition in describing transient by initial dynamic from the rest of dynamics (Fig. 3). Considering that the choice of values for $T_{\mathrm{r}}$ and $c$ affects the accuracy, in this paper the following values have been selected: $T_{\mathrm{r}}=0.5\left(T_{\mathrm{i}}+T_{\mathrm{e}}\right), c=1$.

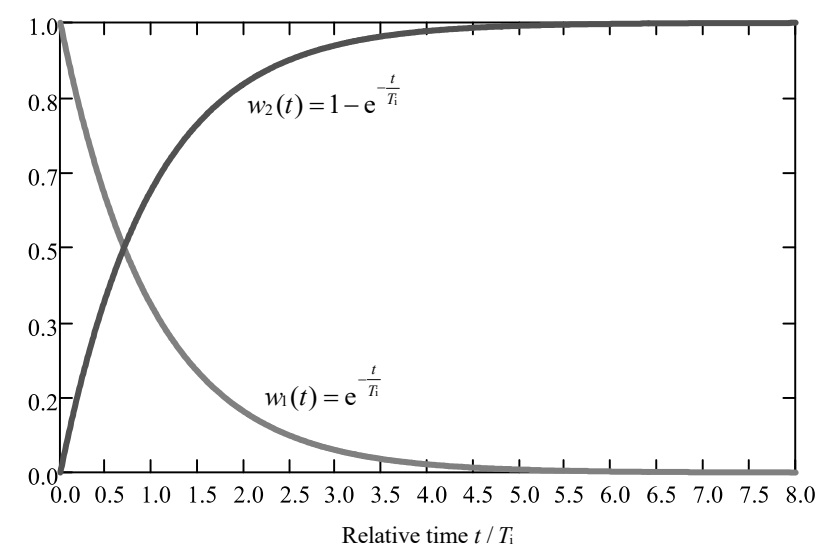

Figure 3. Graph of the functions $w_{1}(t)=\exp \left(-t / T_{\mathrm{i}}\right)$ and $w_{2}(t)=1-\exp \left(-t / T_{\mathrm{i}}\right)$ over the interval $[0$, $+8 T i]$. 
These values will provide an excellent description of the initial part of the transient and a good description of its middle part. This is important because these parts are the most energetic.

\subsection{Generalization}

Based on the previous considerations, a general set of equations describing the voltage and current transients in the case of the fast charging/discharging regime for the voltage dependent SC model can be given as follows:

$$
\begin{gathered}
u_{C}^{\prime}=u_{C}^{\prime}(\infty)-\Delta U_{\max }\left[\mathrm{e}^{-\frac{2 t}{T_{\mathrm{i}}}}+\left(1-\mathrm{e}^{-\frac{t}{T_{\mathrm{i}}}}\right) \mathrm{e}^{-\frac{t}{T_{\mathrm{r}}}}\right] \\
i_{C}=I_{\max }\left[\mathrm{e}^{-\frac{2 t}{T_{\mathrm{i}}}}+\left(1-\mathrm{e}^{-\frac{t}{T_{\mathrm{i}}}}\right) \mathrm{e}^{-\frac{t}{T_{\mathrm{r}}}}\right] \\
\Delta U_{\max }=u_{C}^{\prime}(\infty)-u_{C}^{\prime}(0) \\
I_{\max }=\frac{\Delta U_{\max }}{R_{\mathrm{eqv}}} \\
T_{\mathrm{e}}=T\left(u_{C}^{\prime}(0)\right)=R_{\mathrm{eqv}}\left(C_{0}+2 \frac{C_{\mathrm{r}}-C_{0}}{U_{\mathrm{r}}} u_{C}^{\prime}(0)\right) \\
=R_{\mathrm{eqv}}\left(C_{0}+2 \frac{C_{\mathrm{r}}-C_{0}}{U_{\mathrm{r}}} u_{C}^{\prime}(\infty)\right) \\
T_{\mathrm{r}}=\frac{1}{2}\left(T_{\mathrm{i}}+T_{\mathrm{e}}\right)
\end{gathered}
$$

\section{Energy-related physical quantities}

For a complete transient description, it is necessary to know energy-related physical quantities. If the current is described with an expression that can be integrated, then some of the energy-related physical quantities can be obtained directly by its integration. Otherwise, they must be determined in a different way, as shown in this section. Both approaches are equally valid.

The accumulated energy in SC from the moment $t_{0}$ to the moment $t$ is defined by expression

$$
E_{C}\left(t_{0}, t\right)=\int_{t_{0}}^{t} u_{C}^{\prime} i_{C} \mathrm{~d} t
$$

Substituting (9) into (38) gives

$$
E_{C}\left(t_{0}, t\right)=\int_{t_{0}}^{t}\left(u_{C}^{\prime} \cdot\left(C_{0}+2 \cdot k \cdot u_{C}^{\prime}\right) \frac{\mathrm{d} u_{C}^{\prime}}{\mathrm{d} t}\right) \mathrm{d} t
$$

Minor editing of the previous expression gives

$$
E_{C}\left(t_{0}, t\right)=\int_{t_{0}}^{t}\left(C_{0} \cdot u_{C}^{\prime}+2 \cdot k \cdot u_{C}^{\prime 2}\right) \mathrm{d} u_{C}^{\prime}
$$

Integrating (40) within the limits $t_{0}$ and $t$ gives

$$
\begin{aligned}
E_{C}\left(t_{0}, t\right) & =\frac{1}{2} C_{0}\left(u_{C}^{2}(t)-u_{C}^{2}\left(t_{0}\right)\right)+ \\
& +\frac{2}{3} k\left(u_{C}^{3}(t)-u_{C}^{3}\left(t_{0}\right)\right)
\end{aligned}
$$

Substituting (2) into previous expression gives

$$
\begin{aligned}
E_{C}\left(t_{0}, t\right)= & \frac{1}{2} C_{0}\left[u_{C}^{2}(t)-u_{C}^{2}\left(t_{0}\right)\right]+ \\
& +\frac{2}{3} \frac{C_{\mathrm{r}}-C_{0}}{U_{\mathrm{r}}}\left[u_{C}^{3}(t)-u_{C}^{3}\left(t_{0}\right)\right] .
\end{aligned}
$$

Energy delivered by the source from the moment $t_{0}$ until the moment $t$ is given by

$$
E_{\text {source }}\left(t_{0}, t\right)=\int_{t_{0}}^{t}\left(E \cdot i_{C}\right) \mathrm{d} t
$$

Substituting (9) into previous expression gives

$$
E_{\text {source }}\left(t_{0}, t\right)=E \cdot \int_{t_{0}}^{t}\left(\left(C_{0}+2 \cdot k \cdot u_{C}^{\prime}\right) \frac{\mathrm{d} u_{C}^{\prime}}{\mathrm{d} t}\right) \mathrm{d} t
$$

Minor editing of the previous expression gives

$$
E_{\text {source }}\left(t_{0}, t\right)=E \cdot \int_{t_{0}}^{t}\left(C_{0}+2 \cdot k \cdot u_{C}^{\prime}\right) \mathrm{d} u_{C}^{\prime}
$$

Integrating (45) within the limits $t_{0}$ and $t$ gives 


$$
\begin{aligned}
E_{\text {source }}\left(t_{0}, t\right)= & E \cdot C_{0}\left(u_{C}^{\prime}(t)-u_{C}^{\prime}\left(t_{0}\right)\right)+ \\
& +2 \cdot E \cdot \frac{1}{2} k\left(u_{C}^{2}(t)-u_{C}^{2}\left(t_{0}\right)\right)
\end{aligned}
$$

Substituting (2) into previous expression gives

$$
\begin{aligned}
E_{\text {source }}\left(t_{0}, t\right) & =E \cdot C_{0}\left(u_{C}^{\prime}(t)-u_{C}^{\prime}\left(t_{0}\right)\right)+ \\
& +E \cdot \frac{C_{\mathrm{r}}-C_{0}}{U_{\mathrm{r}}}\left(u_{C}^{2}(t)-u_{C}^{2}\left(t_{0}\right)\right)
\end{aligned}
$$

Total energy loss in the circuit from the moment $t_{0}$ until the moment $t$ is given by

$$
E_{\text {total losses }}\left(t_{0}, t\right)=\left(R+R_{\mathrm{w}}+R_{\mathrm{i}}+R_{0}\right) \int_{t_{0}}^{t} i_{C}^{2} \mathrm{~d} t
$$

This energy corresponds to the difference between the energy supplied by the source and the energy stored in SC

$$
E_{\text {total losses }}\left(t_{0}, t\right)=E_{\text {source }}\left(t_{0}, t\right)-E_{C}\left(t_{0}, t\right)
$$

Energy loss in SC from the moment $t_{0}$ until the moment $t$ is given by

$$
E_{\text {losses SC }}\left(t_{0}, t\right)=\left(R_{\mathrm{i}}+R_{0}\right) \int_{t_{0}}^{t} i_{C}^{2} \mathrm{~d} t
$$

By dividing (50) with (48) and after simplifying integrals in the numerator and denominator gives

$$
\frac{E_{\text {losses SC }}\left(t_{0}, t\right)}{E_{\text {total losses }}\left(t_{0}, t\right)}=\frac{R_{\mathrm{i}}+R_{0}}{R+R_{\mathrm{w}}+R_{\mathrm{i}}+R_{0}}
$$

Substituting (49) into previous expression gives

$$
\begin{aligned}
E_{\text {losses SC }}\left(t_{0}, t\right)= & \frac{R_{\mathrm{i}}+R_{0}}{R+R_{\mathrm{w}}+R_{\mathrm{i}}+R_{0}} . \\
& \cdot\left(E_{\text {source }}\left(t_{0}, t\right)-E_{C}\left(t_{0}, t\right)\right)
\end{aligned}
$$

where $E_{C}\left(t_{0}, t\right)$ and $E_{\text {source }}\left(t_{0}, t\right)$ are determined with expressions (42) and (47).

This shows that it is not necessary to integrate the current or its squares to determine $E_{\text {loses SC }}\left(t_{0}, t\right)$, $E_{\text {source }}\left(t_{0}, t\right)$ and $E_{C}\left(t_{0}, t\right)$. It is enough to know the initial SC voltage $u_{C}^{\prime}\left(t_{0}\right)$ and voltage in the moment $t$, i.e. $u_{C}^{\prime}(t)$. Both of them are determined by the proposed expression for SC voltage.

\section{Results and Discussion}

For a better understanding of the need for a more accurate description of the voltage dependent SC model, an error that occurs in the worst-case scenario must be analyzed. Such cases occur when the voltage of the SC changes in the range from zero to the rated voltage and vice versa. The first analysis was conducted for the approximations traditionally taken in engineering. The second analysis was conducted for the proposed expressions. In both groups of analysis, the exact solution is considered to be the one obtained by the numerical solution of the NDE (13) that describes the transient phenomenon. Readymade software routines "Odesolve" within the software package "Mathcad 14" were used for solving the NDE (13). All simulations were carried out for the initial uncharged SC $\left(u_{C}^{\prime}(0)\right)$, which is charged up to the rated voltage $u_{c}^{\prime}(\infty)=U_{\mathrm{r}}$. According to [20]. supercapacitors have simple models with which we have used a different approach. In order for the presented results to be generalized, they are not expressed using numerical values of circuit parameters. This also facilitates the analysis of the presented results.

\subsection{Traditional approximations in engineering}

Figs. 4-7 demonstrate the error made in the description of voltages and current of SC that occurred by ignoring voltage dependent capacitance in the situation when this capacitance changes by 50 $\%$, which corresponds to $T_{\mathrm{e}}=2 T_{\mathrm{i}}$. Two common cases were used in engineering. The first (Figs. 4 and 5 ), when voltage and current are described by the approximate expressions (53) and (54) which describe the dynamics of whole transient using initial time constant

$$
\begin{gathered}
u_{C, \text { ap }}^{\prime}=-\Delta U_{\max } \cdot \mathrm{e}^{-\frac{t}{T_{\mathrm{i}}}}+u_{C}^{\prime}(\infty) \\
i_{C, \text { ap }}=I_{\max } \cdot \mathrm{e}^{-\frac{t}{T_{\mathrm{i}}}}
\end{gathered}
$$

The second case (Figs. 6 and 7), when voltage and current are described by the approximate expressions (55) and (56), which describe the dynamics of the whole transient using the average time constant. 


$$
\begin{gathered}
u_{C, \text { ap }}^{\prime}=-\Delta U_{\max } \cdot \mathrm{e}^{-\frac{t}{0.5\left(T_{\mathrm{i}}+T_{\mathrm{e}}\right)}}+u_{C}^{\prime}(\infty) \\
i_{C, \text { ap }}=I_{\max } \cdot \mathrm{e}^{-\frac{t}{0.5\left(T_{\mathrm{i}}+T_{\mathrm{e}}\right)}}
\end{gathered}
$$

\subsection{Proposed approximations}

The previous analysis provided an insight into the error made in engineering by neglecting the SCs voltage-dependent capacitance. This new one, by comparing the proposed expressions with the exact solution, will give insight into progress that has been made in reducing the error in the description of transient in relation to approximations used in engineering.

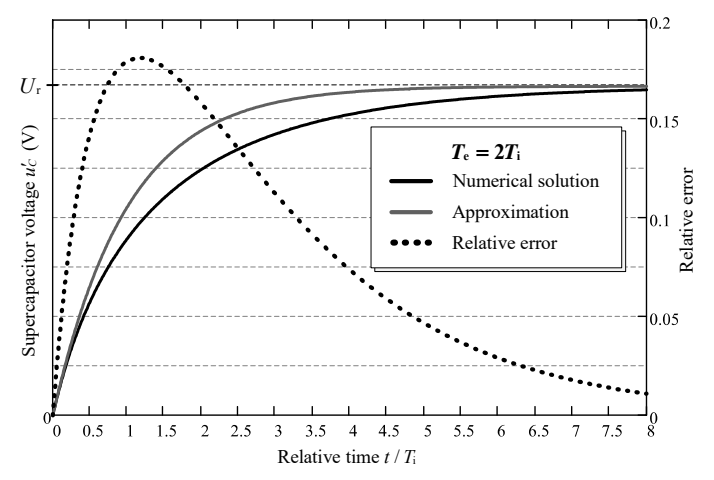

Figure 4. Graph of the SC voltages $u_{C}^{\prime}$ obtained by numerically solving nonlinear differential equation, its approximation, and corresponding error for the case where $T_{\mathrm{e}}=2 T_{\mathrm{i}}$ using initial time constant.

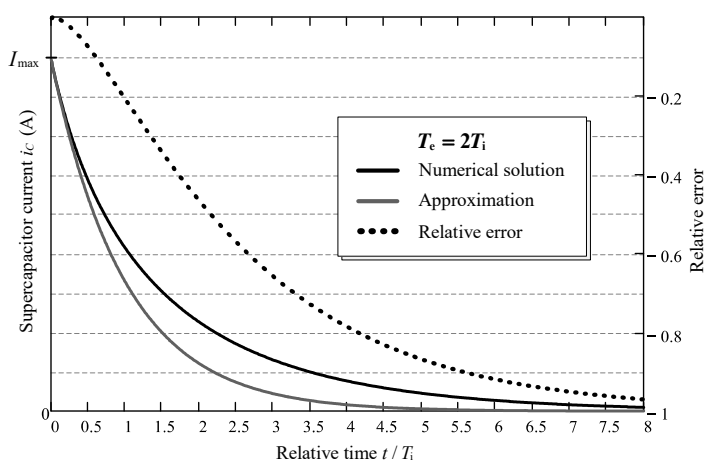

Figure 5. Graph of the SC current $i_{C}$ obtained by numerically solving nonlinear differential equation, its approximation, and corresponding error for the case where $T_{\mathrm{e}}=2 T_{\mathrm{i}}$ using initial time constant.

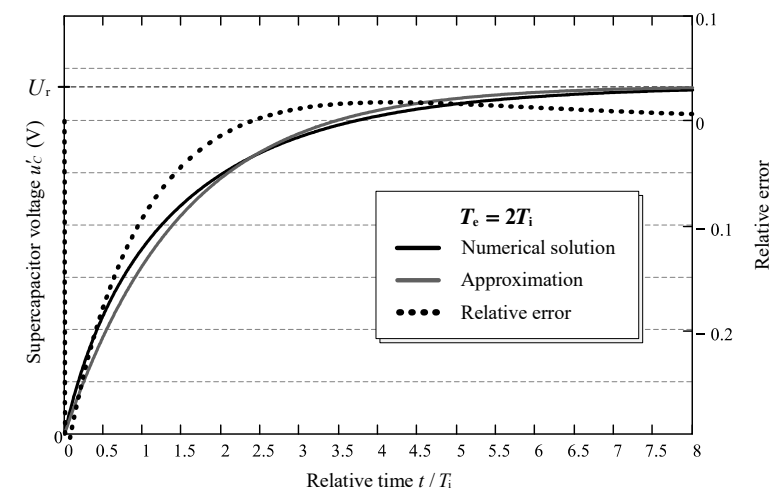

Figure 6. Graph of the SC voltages $u_{C}^{\prime}$ obtained by numerically solving nonlinear differential equation, its approximation, and corresponding error for the case where $T_{\mathrm{e}}=2 T_{\mathrm{i}}$ using average time constant.

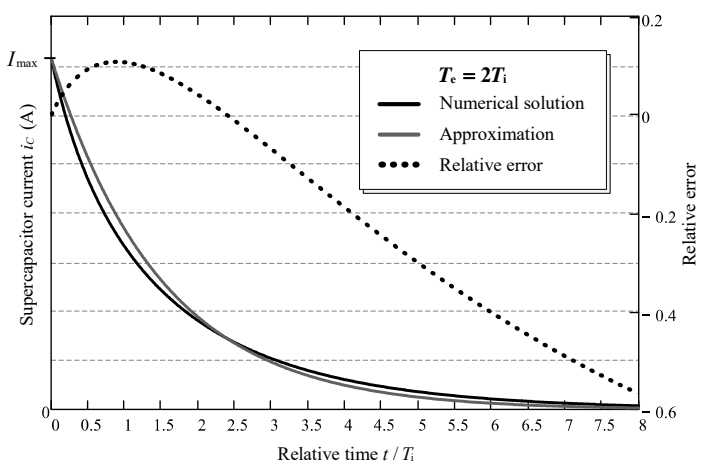

Figure 7. Graph of the SC current $i_{C}$ obtained by numerically solving nonlinear differential equation, its approximation, and corresponding error for the case where $T_{\mathrm{e}}=2 T_{\mathrm{i}}$ using average time constant.

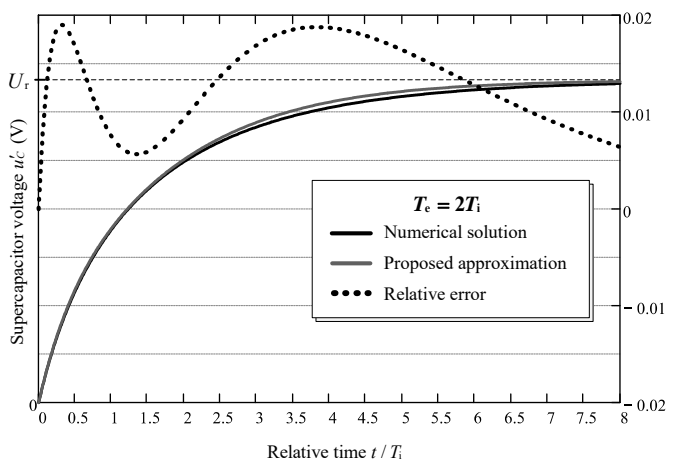

Figure 8. Graph of the SC voltages $u_{C}^{\prime}$ obtained by numerically solving nonlinear differential equation, proposed approximation, and corresponding error for the case where $T_{\mathrm{e}}=2 T_{\mathrm{i}}$ using intuitive approach. 


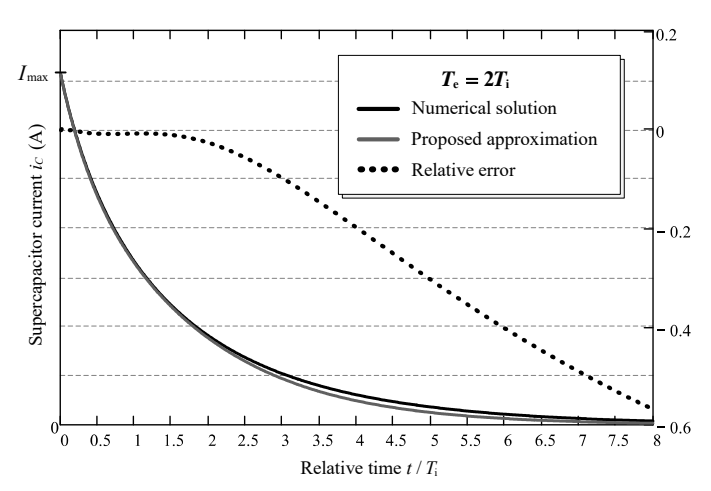

Figure 9. Graph of the SC current $i_{C}$ obtained by numerically solving nonlinear differential equation, proposed approximation, and corresponding error for the case where $T_{\mathrm{e}}=2 T_{\mathrm{i}}$ using intuitive approach .

\subsection{Discussion}

By comparing the waveforms of SCs voltage obtained by traditional approximations in engineering (Figs. 4 and 5) and obtained by proposed expressions (Fig. 8), a dramatic improvement is evident in the description of the waveform appearance in the first half of the transient. Also, the error was reduced from $18 \%$ (Fig. 4) to $2.75 \%$ (Fig. 8 ). This improvement is consistent with previous observations [16].

The second half of the transient phenomenon is also better described, but not so dramatically, as expected, because the emphasis was on improving the initial transient part.

In Fig. 6 a sudden jump of the error from $0 \%$ to $-30 \%$ at the moment $t=0$ can be observed. The reason for this is that according to the expression (55), the voltage is matched for the moment $t=0$ but transient dynamics are not matched because the dynamics of the transient are described by the average time constant (Eq. 55), but in the initial part its dynamics should be described with an initial time constant.

By comparing the waveforms of SCs current obtained by traditional approximations in engineering (Figs. 5 and 7) and obtained by the proposed expressions (Fig. 9), a dramatic improvement in the description of the waveform appearance in the first half of the transient is also evident.

Although the proposed expressions enhance the description of the last part of the transient to some extent, there is a noticeable error in the case of current descriptions. This error at the end of the transient should be interpreted with caution. Although energetically the transient is mostly completed and the current has almost dropped to zero, a relatively high error ( $-55 \%$ on Fig. 9) is observed.

The relatively high error (-55\% on Fig. 9) before the end of transient is the result of the definition of the relative error given by $\delta p=\left(f_{\text {obtained }}-f_{\text {true }}\right) / f_{\text {true }}$. Namely, in this case due to the small currents, both in numerator and denominator, the numerator is divided by a small denominator value and the error has a significant value, although the amount of current is of no practical significance. This part of the transient is dominated by currents caused by dielectric absorption which are not included in the presented model. These currents are larger than the current which remains on the basis of the proposed expressions or obtained by numerically solving the NDE. For these reasons, it is not necessary to insist on improving the proposed expressions.

According to the expression (26), which is further confirmed by Figs. 4-9, voltage-dependent capacitance has twice the greater influence on the time constant than the voltage independent capacitance.

Although the error, in the worst-case scenario, when the proposed expressions are not applied may seem acceptable, that is not the case. The other physical quantities, such as stored energy in SC, energy losses in $\mathrm{SC}$ and circuit depend on the second and the third power SCs voltage (Equations 42, 47, 52). For this reason, the $\mathrm{SC}$ voltage error will propagate in determining these physical quantities. This is an additional reason to use the proposed expressions.

\section{Conclusion}

The article clearly presents and provides a detailed theory with excerpts from all physical quantities required for the description of voltage and current transients in voltage dependent SC model. The validity of the proposed expressions is confirmed by error analysis and visual inspection of the voltage and current waveforms obtained by the proposed model compared to those obtained by the numerical solution of the nonlinear differential equation that describe transient. The presented expressions are fully applicable to all types of supercapacitors and all levels of variations of capacitance. They are applicable both to the charging and discharging processes of the SC. Compared to the traditionally used approximations in engineering, the proposed expressions dramatically improve the description of voltage and current transients in the first half of the transient, especially its initial part and, to some 
extent, its last part. The proposed expressions are simple, elegant, and easy to use.

\section{References}

[1] Yu, A., Chabot, V., Zhang, J.: Electrochemical Supercapacitors for Energy Storage and Delivery: Fundamentals and Applications (Electrochemical Energy Storage and Conversion), CRC Press, 1 edition, 2013.

[2] Conway, B. E.: Electrochemical Supercap.: Scientific Fundamentals and Technological Applications, Springer Science \& Business Media, 2013.

[3] Beguin, F., Frackowiak, E., Lu, M., Supercapacitors: Materials, Systems, and Applications, Wiley-VCH, 1 edition, 2013.

[4] Beguin, F., Frackowiak, E.: Carbons for Electrochemical Energy Storage and Conversion Systems, CRC Press, 1 edition, 2009.

[5] Kötz, R., Carlen, M.: Principles and applications of electrochmeical capcitors, Electrochimica Acta, Volume 45, Issues 15-16, 3 2000, 2483-2498.

[6] Deshpande, R. P.: Ultracapacitors, McGrawHill Education, 2014.

[7] Miller, J. M.: Ultracapacitor Applications, The Institution of Engineering and Technology, 2011.

[8] Logerais, P.O., Camara, M.A., Riou, O., Djellad, A., Omeiri, A., Delaleux, F., Durastanti, J.F.: Modeling of a supercapacitor with a multibranch circuit, International Journal of Hydrogen Energy, 40 (2015) 39, 13725-13736.

[9] Funaki, T., Hikihara, T.: Characterization and Modeling of the Voltage Dependency of Capacitance and Impedance Frequency Characteristics of Packed EDLCs, IEEE Transactions on Power Electronics, 23, 15181525.

[10] Sarwar, W., Marinescu, M., Green, N., Taylor, N., Offer, G.: Electrochemical double layer capacitor electro-thermal modelling, Journal of Energy Storage, 5, (2016), 10-24.
[11] Saha, P., Khanra, M.: Equivalent Circuit Model of Supercapacitor for Self-Discharge Analysis A Comparative Study, International conference on Signal Processing, Communication, Power and Embedded System (SCOPES)-2016, Paralakhemundi, 2016, 1381-1386.

[12] Faranda, R., Gallina, M., Son, D. T.: A new simplified model of double-layer capacitor, International Conference on Clean Electrical Power, Capri, 2007, 706-710.

[13] Zubieta, L. E.: Characterization of double-layer capacitors for Power electronics applications, A thesis for the degree of master of applied science, University of Toronto, 1997.

[14] Fletcher, S., Black, V. J. and Kirkpatrick, I.: A universal equivalent circuit for carbon-based supercapacitors, Journal of Solid State Electrochemistry, 18 (2014), 5, 1377-1387.

[15] Dougal, R. A., Gao, L., Liu, S.: Ultracapacitor model with automatic order selection and capacity scaling for dynamic system simulation, Journal of Power Sources 126 (2004), 250-257.

[16] Rafik, F., Gualous, H., Gallay, R., Crausaz, A., Berthon, A.: Frequency, thermal and voltage supercapacitor characterization and modeling, Journal of Power Sources, 165 (2007), 2, 928934.

[17] Grama, A., Petrcus, D., Borza, P., Grama, L.: Experimental Determination of Equivalent Series Resistance of a Supercapacitor, 32nd International Spring Seminar on Electronics Technology, (ISSE 2009), Brno, Czech Republic, 2009, 1-4.

[18] Gualous, H., Bouquain, D., Berthon, A., Kauffmann, J. M.: Experimental study of supercapacitor serial resistance and capacitance variations with temperature, Journal of Power Sources, 123 (2003), 1, 86-93.

[19] Harris, J. W., Stocker, H.: Handbook of Mathematics and Computational Science, 1998, Springer - Verlag, New York, Inc.

[20] Sladić, S., Poljak, A., Bulić, N.: Towards the new energy storage system for conventional cars, Engineering Review, 38 (2018), 3, 261267. 